\title{
Commentary
}

\section{Pain services and prescription of opioid drugs in Ghana}

\author{
Ghana Med J 2016;50(2):62 DOI: http://dx.doi.org/10.4314/gmj.v50i2.1
}

Pain is probably the commonest symptom that sends patients to seek medical attention. Many people in Ghana accept pain as part of their normal lives when it is mild or moderate in severity. For treatment, they go to community pharmacies or chemical shops to purchase pain relievers. Some individuals will procure substances or medications from friends and relatives for the relief of their pain.

In developed countries, the Primary Health Clinics have reasonable provisions and guidelines to manage most types and levels of pain in the community. Acute Pain Services (APS) have evolved in most hospitals to take care of post-operative pain in surgery, labour pain in obstetrics and other areas of medical and surgical of practice as well as handling referrals from the primary health clinics.

Multi-disciplinary Pain Teams involving specialists like anaesthetists, surgeons, physicians, pharmacists, physiotherapists, psychologists, palliative care, radiologists have also evolved to manage Chronic Pain Syndromes (CPS). This is a departure from the single specialist practice of old as these syndromes may be very complex. Different presentations of pain like nociceptive pain, neuropathic pain, cancer pain, central post stroke pain, phantom pain and referred pain are all now better understood but remain difficult to treat without a multidisciplinary approach and adequate resources and commitment.

It is therefore no longer acceptable to dismiss pain as "psychogenic" and send patients to psychiatrists and faith healers if no immediate cause is found or when patients do not easily respond to initial treatment. It is the patient's right and not a privilege to have his pain well investigated and treated. ${ }^{1}$

Ghana now has four well-established Teaching Hospitals and over 261 public health institutions with doctors, theatres and laboratory facilities to handle a wide variety of surgical, obstetric and medical adult and children's' conditions with moderate and severe pain. ${ }^{2}$ The authors are not aware of a single well-established Acute Pain Service or any Multi-disciplinary Chronic Pain Service in Ghana. Seventy-two out of the 265 districts $(27 \%)$ in Ghana do not have hospitals with operating theatre and laboratory services but it is assumed every district will have pharmacists and over the counter medicines shops ((GHS 2015: Updated unpublished list of
Government and Public Health Facilities with functional Operating Theatres. Accra).

The Food and Drugs Authority, The Ministry of Health and the National Narcotics Control Board should come out with clear guidelines and information for the procurement, prescription and use of opioids in Ghana. There are limited number of audits and observational studies on pain management emanating from surgeons and nurses but large controlled randomized studies are lacking in Ghana. We therefore do not have enough reliable data to formulate national pain policies on the procurement, prescription and use of opioid and nonopioid for pain management.

We welcome the article in this issue (page 64) on the prescribing of opioids by physicians in Nigeria. Although it is not a randomized large study, it gives us an insight into the training and attitudes of doctors in prescribing opioids in our sub-region. Pain services, both acute and chronic, will need to be set up in the teaching and other large hospitals to research and develop protocols and guidelines for the management of all types of pain in Ghana will be a good starting point.

Nurses, pharmacists, basic scientists, physiotherapists, surgeons, physicians, radiologists and others should be encouraged to go into research and management of pain. Programs and facilities related to pain should be adequately funded and resourced at governmental and institutional levels in order to make any real impact on our society.

\section{Dr Frank K Boni, FFARCS(I) \\ E-mail: fkboni@gmail.com \\ and}

Dr Barima A Afrane PhD

E-mail: barimafrane@gmail.com

Department of Pharmacy Practice and Clinical Pharmacy, School of Pharmacy, University of Ghana, Legon, Ghana.

\section{REFERENCES}

1. Brennan F, Carr DB, Cousins M. Pain management: a fundamental human right. Anesth. Analg. 2007 Jul;105 (1):205-21

2. National Assessment for Emergency Obstetric and Neonatal Care. Facilities for referrals. Chapter 5. Ministry of Health/Ghana Health Service, Government of Ghana. Accra, 2011 